\title{
CLOSURE THEOREMS WITH APPLICATIONS TO ENTIRE FUNCTIONS WITH GAPS
}

\author{
BY \\ J. M. ANDERSON AND K. G. BINMORE
}

\begin{abstract}
In this paper we consider questions of completeness for spaces of continuous functions on a half line which satisfy appropriate growth conditions. The results obtained have consequences in the theory of entire functions with gap power series. In particular we show that, under an appropriate gap hypothesis, the rate of growth of an entire function in the whole plane is determined by its rate of growth along any given ray.
\end{abstract}

1. Introduction. We consider nonconstant entire functions $f(z)$ which have gap power series expansions-i.e. expansions of the form

$$
f(z)=\sum_{n=0}^{\infty} a_{n} z^{n}
$$

in which $a_{n}=0(n \notin \Lambda)$, where $\Lambda$ is a given set of positive integers. The maximum modulus of $f(z)$ on the circle $|z|=r$ will be denoted by $M(r)=M(r, f)$. As is well known, the function

$$
m(s)=m(s, f)=\log M\left(e^{s}\right)
$$

is a convex function of $s$.

The growth of $M(r, f)$ will be measured by comparing it with that of a given function $H(r)$. We shall always suppose that $H(r)$ is a positive increasing function defined for $0 \leqq r<\infty$ and that

$$
h(s)=\log H\left(e^{s}\right)
$$

is a convex function of $s$. We shall assume, in fact, that $h(s)$ has a positive second derivative at every point. This simplifies some of the proofs and is not an important restriction. We shall also insist that, for each integer $n$,

$$
r^{-n} H(r) \rightarrow+\infty \quad(r \rightarrow+\infty) .
$$

This amounts to excluding the case of polynomial growth (see Theorem A of §2).

The set $\Lambda$ is to be thought of as being lacunary in an appropriate sense. Our theorems show that, under such an hypothesis, the behaviour of $M(r, f)$ is deter-

Received by the editors August 31, 1970 and, in revised form, December 18, 1970.

AMS 1969 subject classifications. Primary 3057, 3024; Secondary 4160.

Key words and phrases. Banach space, entire function, closure, approximation, power series.

Copyright (C) 1971, American Mathematical Society 
mined by the behaviour of $f(z)$ along any given ray emanating from the origin. We shall always suppose such a ray to be the positive real axis, but this, of course, involves no loss of generality. Our theory applies to functions with an arbitrary rate of growth, but, as some of our theorems are known in the case of functions of finite order-i.e. functions for which

$$
\rho=\limsup _{r \rightarrow \infty} \frac{\log \log M(r)}{\log r}<\infty,
$$

it may be advantageous to think of the functions $H(r)$ as growing very rapidly indeed.

Using the methods of [1], the following theorem is easily proved.

THEOREM 1. Suppose that $\left(^{(}\right)$

$$
\sum \lambda_{n}^{-1}<\infty
$$

Then the hypothesis

$$
|f(x)| \leqq H(x) \quad(0 \leqq x<\infty)
$$

implies that, for each $\sigma>1$, there is a constant $A$ such that

$$
M(r, f) \leqq A H(\sigma r) \quad(0 \leqq r<\infty) .
$$

This extends a theorem of Gaier [6]. In Gaier's theorem, condition (1.6) is replaced by the hypothesis that $f$ has finite order $\rho$ and mean type $\tau$ along a ray, and (1.7) is replaced by the conclusion that $f$ has order $\rho$ and type $\tau$ in the whole plane.

Our initial aim in writing this paper is to examine the extent to which the gap condition (1.5) can be relaxed if one takes into account the rate of growth of $f(z)$. This question is discussed in $\S 2$. The arguments used depend very strongly on the work of Malliavin [11] concerning the completeness of systems of monomials $\left\{x^{\lambda_{n}}\right\}$ in a weighted Banach space.

Let $S_{H}$ denote the Banach space consisting of all continuous functions $f(x)$ on $[0, \infty)$ for which $f(0)=0$ and which satisfy $\lim _{x \rightarrow+\infty}|f(x) / H(x)|=0$. As norm in the space we use

$$
\|f\|_{H}=\max _{x \geqq 0}|f(x) / H(x)| .
$$

Let $V$ denote the linear manifold consisting of all finite linear combinations of the monomials $\left\{x^{\lambda_{n}}\right\}$. In view of (1.4), $V$ is a subset of $S_{H}$. Malliavin gives a necessary and sufficient condition that $V$ be dense in $S_{H}$-i.e. that any $f \in S_{H}$ may be approximated in the $\|\cdot\|_{H}$ norm by linear combinations of the $\left\{x^{\lambda_{n}}\right\}$. A question which Malliavin does not consider, but which is of importance for our application of his work, is the following:

(1) Where the symbol $\sum$ appears with no indication of the range of summation it will be assumed that the summation is from 1 to $\infty$. 
Question. Suppose that $V$ is not dense in $S_{H}$. Then what is the closure $\bar{V}$ of $V$ in $S_{H}$ ?

An analogous problem concerns the Banach space $C_{0}[0,1]$ consisting of all continuous functions $f(x)$ on $[0,1]$ which satisfy $f(0)=0$, the norm being given by $\|f\|=\max _{0 \leqq x \leqq 1}|f(x)|$. This problem was solved by Clarkson and Erdös [3] (see also Korevaar [8]). It is well known that $V$ is dense in $C_{0}[0,1]$ if and only if

$$
\sum \lambda_{n}^{-1}=\infty \text {. }
$$

If this is not the case (i.e. if $\left.\sum \lambda_{n}^{-1}<\infty\right)$ then the closure of $V$ in $C_{0}[0,1]$ turns out to be a surprisingly small set-namely the set of all $f \in C_{0}[0,1]$ which are the restrictions to $[0,1]$ of functions $f(z)$ which are analytic in the open unit disc with a power series expansion of the form

$$
f(z)=\sum A_{n} z^{\lambda_{n}}
$$

By using this result we may say something about the closure of $V$ in $S_{H}$ in the case when $\sum \lambda_{n}^{-1}<\infty$. If $f(x)$ belongs to the closure of $V$ in $S_{H}$, then, for each $R>0$, the function $f(R x)$ belongs to the closure of $V$ in the space $C_{0}[0,1]$. In view of the result of Clarkson and Erdös mentioned above, we deduce that $f$ is the restriction to the positive real axis of an entire function $f(z)$ with a power series expansion of the form (1.9). Moreover, since $f \in S_{H}$, we have that

$$
f(x)=O(H(x)) \quad(x \rightarrow+\infty) .
$$

On employing Theorem 1, we deduce that, for each $\sigma>1$,

$$
M(r, f)=O(H(\sigma r)) \quad(r \rightarrow+\infty) .
$$

For $0<\sigma<\infty$, let $E_{\sigma}$ denote the set of all entire functions $f(z)$ satisfying (1.9) and (1.10). From the above remarks we have that $\bar{V} \subset E_{\sigma}(\sigma>1)$.

On the other hand, if $f \in E_{\sigma}(\sigma<1)$, then it is easily seen (as in Theorem 4 below) that the partial sums of its power series expansion converge to $f(z)$ in the $\|\cdot\|_{H}$ norm and hence that $f \in \bar{V}$.

If $\sum \lambda_{n}^{-1}<\infty$, we can therefore assert that

$$
E_{\alpha} \subset \bar{V} \subset E_{\beta}
$$

provided that $0<\alpha<1<\beta$. The more difficult case when $\sum \lambda_{n}^{-1}=\infty$ is discussed in $\S 4$. In neither case, however, are we able to give a precise characterisation of the set $\bar{V}$.

To conclude this section we remark that the case $H(x)=e^{x}$ of Malliavin's results had been obtained earlier by Fuchs [5]. The question of noncompleteness in this case has been discussed by Leont'ev [10] and our results of $\$ 4$ complement his to the extent that he deals only with the case of finite order.

2. Growth theorems. We begin with some definitions. As always $h(s)$ is given by (1.3). If $\Lambda$ is a set of positive integers, we introduce the function

$$
L_{h}(x)=\sum_{\lambda_{n}<n(x)} \lambda_{n}^{-1}
$$


and define the quantities $\bar{D}_{h}$ and $\underline{D}_{h}$ by

$$
\bar{D}_{h}=\bar{D}_{h}(\Lambda)=\limsup _{x \rightarrow+\infty} x^{-1} L_{h}(x), \quad \underline{D}_{h}=\underline{D}_{h}(\Lambda)=\liminf _{x \rightarrow+\infty} x^{-1} L_{h}(x) .
$$

We shall refer to $\bar{D}_{h}$ and $\underline{D}_{h}$ as the upper and lower $h$-densities of the set $\Lambda$. Note, however, that these numbers may exceed one.

The set $\Lambda$ will be said to be $h$-measurable if $\bar{D}_{h}=\underline{D}_{h}$, in which case the common value will be denoted by $D_{h}$. We may call $D_{h}$ the $h$-density of the set $\Lambda$. Following Kahane [7], we may associate with $D_{h}$ a "densité extérieure" $D_{h}^{*}$. This number is defined by

$$
D_{h}^{*}=D_{h}^{*}(\Lambda)=\inf D_{h}(\mu)
$$

where the infimum is extended over all $h$-measurable sets $\mu$ which contain the set $\Lambda$. To be consistent with what seems to be the English speaking usage, we shall refer to $D_{h}^{*}$ as the maximum $h$-density of the set $\Lambda$. The following inequality clearly holds for all $h$ and $\Lambda$ :

$$
\underline{D}_{h} \leqq \bar{D}_{h} \leqq D_{h}^{*}
$$

The case when $h$ is the exponential function has been considered, for example, by Edrei [4] and by Malliavin [11]. They refer to the densities involved as "logarithmic". We remark that the "maximum logarithmic density" $D_{\text {exp }}^{*}(\Lambda)$ of a set $\Lambda$ admits the following alternative definition-namely

$$
D_{\text {exp }}^{*}(\Lambda)=\lim _{\xi \rightarrow 1-} \limsup _{x \rightarrow+\infty}\left\{\frac{L(x)-L\left(x^{\xi}\right)}{(1-\xi) \log x}\right\}
$$

where $L(x)=\sum_{\lambda_{n}<x} \lambda_{n}^{-1}$.

Suppose now that $f(z)$ is an entire function and that $m(s)=m(s, f)$ is given by (1.2). We shall say that $f(z)$ has $h$-order $\rho$ (in the whole plane) if

$$
\rho=\limsup _{s \rightarrow \infty} \frac{h^{-1}(m(s))}{s} .
$$

The $h$-order reduces to the usual notion of order in the case when $h$ is the exponential function. We shall also speak of a function having $h$-order $\rho$ "along a ray" or "in an angle", the definitions being the obvious ones.

Two increasing convex functions, $h(s)$ and $k(s)$, will be said to be comparable if the limit

$$
l=\lim _{s \rightarrow \infty} \frac{h^{-1}(k(s))}{s}
$$

exists. Here $0 \leqq l \leqq \infty$. If $0<l<\infty$, then an entire function $f(z)$ has $h$-order $\rho$ if and only if it has $k$-order $\rho l^{-1}$.

In a number of our theorems, we require that the function $h(s)$, in addition to satisfying the requirements of $\S 1$, have the property that $\log h(s)$ is a convex 
function of $s$. This implies that $(\log h(s)) / s \rightarrow m(s \rightarrow+\infty)$ for some $m(0<m \leqq \infty)$. The interesting case, of course, is that when $m=\infty$, since, if $m<\infty$, it follows from the above remarks that $f(z)$ has $h$-order $\rho$ if and only if it has (ordinary) order $m \rho$.

With these definitions we now state

THEOREM 2. Suppose that $\log h(s)$ is a convex function of $s$ and that $k(s)$ is a convex function of $s$ which is comparable with $h(s)$. Let $f(z)$, given by (1.1), be an entire function with h-order at most $\rho<\infty$ and suppose that

$$
D_{h}^{*}(\Lambda)<1 / 2 \rho .
$$

Then the hypothesis that $f(z)$ has $k$-order $\sigma<\rho$ along a ray implies that $f(z)$ has $k$-order $\sigma$ in the whole plane.

This implies that an entire function $f(z)$ satisfying the conditions of Theorem 2 has the same $h$-order along every ray as it does in the whole plane. On taking $h(s)$ $=k(s)=\exp s$, we obtain the following corollary (see Kövari [9, Theorem 1] for a somewhat similar result).

COROLlaRY 1. Let $f(z)$ have order at most $\rho<\infty$ and suppose that

$$
D_{\text {exp }}^{*}(\Lambda)<1 / 2 \rho .
$$

Then the hypothesis that $f(z)$ has order $\sigma<\rho$ along a ray implies that $f(z)$ has order $\sigma$ in the whole plane.

The question arises as to whether or not Theorem 2 is "best possible". Since $k(s)$ is convex, we have that $k(s) / s \rightarrow \gamma(s \rightarrow+\infty)$ where $0 \leqq \gamma \leqq \infty$. If $\gamma<\infty$, the hypothesis that $f$ has $k$-order $\sigma<\infty$ along a ray implies that $f$ has polynomial growth along the ray. This is a situation we have considered elsewhere [2]. In the notation of this section we have

THEOREM A. Let $f(z)$ have h-order $\rho<\infty$ and suppose that

$$
\underline{D}_{h}(\Lambda)<1 / 2 \rho .
$$

Then the hypothesis that $f$ is majorised by some polynomial along a ray implies that $f$ is itself a polynomial.

Conversely, if $\log h(s)$ is convex, then, given any set of positive integers $\Lambda$ for which $\underline{D}_{h}(\Lambda) \geqq 1 / 2 \rho$, there exists a transcendental entire function $f(z)$ with a gap power series expansion of the form (1.1) and of h-order at most $\rho$ but such that $f(x) \rightarrow 0(x \rightarrow+\infty)$.

Thus, Theorem 2 and Corollary 1 are best possible in the sense that the number $(2 \rho)^{-1}$ which appears in (2.4) and (2.5) cannot be replaced by any larger constant. One simply applies Theorem A to a set $\Lambda$ for which $\underline{D}_{h}(\Lambda)=D_{h}^{*}(\Lambda)$. However, it seems possible that Theorem 2 remains true if condition (2.4) is replaced by (2.6). We have not been able to settle this question, even in the case when $h(s)$ is the exponential function. 
In addition to considering growth along a ray, one can also consider how the growth in an angle of an entire function with a gap power series expansion affects its growth in the whole plane. We discuss this question in $\$ 7$.

3. Approximation results. We now turn to the Banach space $S_{H}$, and the various other concepts introduced in $\S 1$. We recall that $H(s)$ is a positive increasing function defined for $0 \leqq s<\infty$. In particular, $H(0)>0$. Also $h(s)=\log H\left(e^{s}\right)$ is a convex function of $s$. For the given set $\Lambda=\left\{\lambda_{n}\right\}$ of positive integers we define

$$
\lambda(r)=2 \sum_{\lambda_{n}<r} \lambda_{n}^{-1}
$$

As before, $V$ denotes the set of all finite linear combinations of the monomials $\left\{x^{\lambda}\right\}$.

Malliavin [11] proves the following theorem:

THEOREM B. A necessary and sufficient condition that $V$ be dense in $S_{H}$ is that, for each real constant $a$,

$$
\int^{+\infty} h(\lambda(r)-a) \frac{d r}{r^{2}}=+\infty
$$

The dual space $S_{H}^{\prime}$ of $S_{H}$ is the set of all measures $\mu$ on $[0, \infty)$ for which

$$
\|\mu\|_{H}=\int_{0}^{\infty} H(t)|d \mu(t)|<\infty .
$$

It is well known that a necessary and sufficient condition for $V$ to be dense in $S_{H}$ (i.e. that the sequence $\left\{x^{\lambda_{n}}\right\}$ be closed in $S_{H}$ ) is that the only measure $\mu \in S_{H}^{\prime}$ which satisfies

$$
\int_{0}^{\infty} t^{\lambda_{n}} d \mu(t)=0 \quad(n=1,2, \ldots)
$$

is $\mu \equiv 0$ (i.e. that the sequence $\left\{x^{\lambda}\right\}$ is complete in $S_{H}$ ). To prove the necessity of (3.2) in Theorem B, Malliavin therefore assumes that (3.2) does not hold and constructs a measure $\mu \in S_{H}^{\prime}$ which satisfies (3.3) but does not vanish identically.

Malliavin's construction is very explicit. If (3.2) does not hold, the measure $\mu$ is determined by

$$
G(z)=\int_{0}^{\infty} t^{z-1} d \mu(t)=g(z) \prod_{\lambda \in \Lambda}\left(\frac{1-z /(\lambda+1)}{1+z /(\lambda+1)}\right) e^{2 z /(\lambda+1)}
$$

where $g(z)$ satisfies

$$
\log |g(z)|=-\frac{4 x}{\pi} \int_{-\infty}^{\infty} \frac{\phi(t)}{x^{2}+(y-t)^{2}} d t-b x .
$$

In this equation $b$ is a constant and $\phi(t)$ is a certain even function of $t$ which satisfies $\int^{+\infty} \phi(t) d t / t^{2}<\infty$. 
A sequence $\left\{x^{\lambda_{n}}\right\}$ is said to be free in $S_{H}$ if no $x^{\lambda_{k}}$ can be approximated in the $\|\cdot\|_{H}$ norm by linear combinations of the other elements of $\left\{x^{\lambda_{n}}\right\}$. Let $L_{n}$ be the linear functional defined on $V$ which maps each polynomial $P(x)=\sum a_{k} x^{\lambda_{k}}$ onto its $n$th coefficient. That is to say

$$
a_{n}=L_{n}(P) \text {. }
$$

It is easy to prove that $\left\{x^{\lambda_{n}}\right\}$ is free in $S_{H}$ if and only if each of the linear functionals $L_{n}(n=1,2, \ldots)$ is bounded. As a consequence we deduce that a necessary and sufficient condition for the sequence $\left\{x^{\lambda_{n}}\right\}$ to be free in $S_{H}$ is the existence of measures $\mu_{n} \in S_{H}^{\prime}(n=1,2, \ldots)$ with the property that

$$
\begin{aligned}
\int_{0}^{\infty} t^{\lambda_{k}} d \mu_{n}(t) & =1, & & n=k, \\
& =0, & & n \neq k .
\end{aligned}
$$

The sufficiency is immediate. For the necessity, we note that $L_{n}$ may be extended to the whole of $S_{H}$ by the Hahn-Banach theorem and therefore may be represented in the appropriate form.

We now prove a lemma which implies that the sequence $\left\{x^{\lambda_{n}}\right\}$ is free in $S_{H}$ whenever $V$ is not dense in $S_{H}$. The proof of the lemma requires some remarks concerning the properties of convex functions.

Suppose that $g(x)$ is a convex function of $x$ with the property that

$$
g(x) / x \rightarrow+\infty \quad(x \rightarrow+\infty) .
$$

With such a function we may associate another function $\tau_{g}(y)$ defined by

$$
\tau_{g}(y)=\sup _{x}\{x y-g(x)\}
$$

The function $\tau_{g}(y)$ is itself convex and satisfies (3.7). Moreover (see [12, p. 7]), we have that

$$
g(x)=\sup _{y}\left\{y x-\tau_{g}(y)\right\}
$$

Lemma 1. Suppose that $V$ is not dense in $S_{H}$. Then there exist measures $\mu_{n} \in S_{H}^{\prime}$ $(n=1,2,3, \ldots)$ for which

$$
\begin{aligned}
\int_{0}^{\infty} t^{\lambda_{k}} d \mu_{n}(t) & =1, & & n=k, \\
& =0, & & n \neq k,
\end{aligned}
$$

and such that

$$
\left\|\mu_{n}\right\|_{H} \leqq C^{\lambda_{n}} \exp \left\{-2 \lambda_{n} \sum_{k=1}^{n} \lambda_{k}^{-1}\right\},
$$

where $C$ is a constant depending only on the sequence $\Lambda$ and the function $H(x)$. 
Proof. We set $H_{0}(t)=H(t+1)(t \geqq 0)$ and define, for $x \geqq 0$,

$$
M(x)=\max _{t \geqq 0}\left(\frac{t^{x}}{H_{0}(t)}\right)
$$

We now write $H_{1}(t)=t^{4} H_{0}(t)$. Since $V$ is not dense in $S_{H}$ it follows from Theorem B that $V$ is not dense in $S_{H_{1}}$. Hence we may use Malliavin's construction to obtain a nonzero measure $\mu \in S_{H_{1}}^{\prime}$ with the property that

$$
\int_{0}^{\infty} t^{\lambda_{k}} d \mu(t)=0 \quad(k=1,2, \ldots) .
$$

This measure $\mu$ is determined by (3.4). Since $\mu \in S_{H_{1}}^{\prime}$, we have that, for $x \geqq 4$,

$$
\begin{aligned}
\int_{0}^{\infty} t^{x}|d \mu(t)| & =\int_{0}^{\infty} \frac{t^{x}}{H_{1}(t)} H_{1}(t)|d \mu(t)| \\
& \leqq \sup _{t \geqq 0}\left|\frac{t^{x}}{H_{1}(t)}\right| \cdot\|\mu\|_{H_{1}} \leqq K M(x-4),
\end{aligned}
$$

where $K$ is a constant which depends only on the function $H(s)$ and on $\mu$. Since the function $g(z)$ determined by (3.4) has no zeros, the function $G_{n}(z)=\left(z-\lambda_{n}-1\right)^{-1} G(z)$ has no zero at $z=\lambda_{n}+1$. On writing $\lambda=\lambda_{n}$, we conclude that

$$
\begin{aligned}
G_{n}(z) & =\frac{G(z)}{z-\lambda-1} \\
& =\int_{0}^{\infty} \frac{t^{z-1}}{z-\lambda-1} d \mu(t)=\int_{0}^{\infty} \frac{t^{z-\lambda-1}}{z-\lambda-1} \cdot t^{\lambda} d \mu(t) \\
& =\left[\frac{t^{z-\lambda-1}}{z-\lambda-1} \int_{0}^{t} u^{\lambda} d \mu(u)\right]-\int_{0}^{\infty} t^{z-\lambda-2}\left\{\int_{0}^{t} u^{\lambda} d \mu(u)\right\} d t .
\end{aligned}
$$

We now seek to show that the integrated part of this expression vanishes, provided that $x=\mathscr{R} z>1$. Firstly,

$$
\left|t^{z-\lambda-1} \int_{0}^{t} u^{\lambda} d \mu(u)\right| \leqq t^{x-1} \int_{0}^{t}|d \mu(u)| \rightarrow 0 \quad(t \rightarrow 0+) .
$$

Also, since $G(\lambda+1)=0$,

$$
\int_{0}^{t} u^{\lambda} d \mu(u)=-\int_{t}^{\infty} u^{\lambda} d \mu(u)
$$

and hence

$$
\begin{aligned}
\left|t^{z-\lambda-1} \int_{0}^{t} u^{\lambda} d \mu(u)\right| & =\left|t^{z-\lambda-1} \int_{t}^{\infty} u^{\lambda} d \mu(u)\right| \\
& \leqq \max _{u \geq t}\left|\frac{t^{x-1}}{H_{1}(t)}\right| \cdot\|\mu\|_{H_{1}} \rightarrow 0 \quad(t \rightarrow+\infty) .
\end{aligned}
$$

Thus, for $\mathscr{R} z>1$,

$$
G_{n}(z)=\int_{0}^{\infty} t^{z-1} d \nu_{n}(t)
$$


where $\nu_{n}$ is defined by

$$
d \nu_{n}(t)=-\frac{1}{t^{\lambda+1}}\left\{\int_{0}^{t} u^{\lambda} d \mu(u)\right\} d t .
$$

We shall show that, for each integer $n$,

$$
\left\|\nu_{n}\right\|_{H} \leqq A
$$

where $A$ is a constant depending only on $H$ and $\mu$. Assuming this fact for the moment, we define $\mu_{n}$ by

$$
d \mu_{n}(t)=\frac{1}{G_{n}\left(\lambda_{n}+1\right)} d \nu_{n}(t)
$$

We have from (3.9) that

$$
\begin{aligned}
\int_{0}^{\infty} t^{\lambda_{k}} d \mu_{n}(t)=\frac{G_{n}\left(\lambda_{k}+1\right)}{G_{n}\left(\lambda_{n}+1\right)} & =1, & & n=k, \\
& =0, & & n \neq k .
\end{aligned}
$$

Moreover, it is not difficult to deduce from (3.4) that

$$
\left|G_{n}\left(\lambda_{n}+1\right)\right|^{-1} \leqq B^{\lambda_{n}} \exp \left\{-2 \lambda_{n} \sum_{k=1}^{n} \lambda_{k}^{-1}\right\}
$$

where $B$ is a constant which depends only on the sequence $\Lambda$ and on the function $H(s)$. The necessary estimation for the infinite product which appears in (3.4) is given in Fuchs [5]. It follows immediately from this inequality and from (3.11) that

$$
\left\|\mu_{n}\right\|_{H} \leqq A B^{\lambda_{n}} \exp \left\{-2 \lambda_{n} \sum_{k=1}^{n} \lambda_{k}^{-1}\right\} .
$$

It remains to establish (3.11). Now, if $x \leqq \lambda-1$,

$$
\begin{aligned}
\int_{0}^{\infty} t^{x}\left|d \nu_{n}(t)\right| & \leqq \int_{0}^{\infty} t^{x-\lambda-1} d t \int_{0}^{t} u^{\lambda}|d \mu(u)| \\
& =\int_{0}^{\infty} u^{\lambda}|d \mu(u)| \int_{u}^{\infty} t^{x-\lambda-1} d t=\frac{1}{\lambda-x} \int_{0}^{\infty} u^{x}|d \mu(u)| \\
& \leqq K M(x-4) \quad(x \geqq 4) .
\end{aligned}
$$

Only the last inequality above requires $x \geqq 4$, and so we conclude, in particular, that

$$
\int_{0}^{\infty}\left|d v_{n}(t)\right| \leqq \int_{0}^{\infty}|d \mu(t)|
$$

for each integer $n$, the integrals existing since $H(0)>0$.

If $x \geqq \lambda+1$, 


$$
\begin{aligned}
\int_{0}^{\infty} t^{x}\left|d v_{n}(t)\right| & =\int_{0}^{\infty} t^{x-\lambda-1} d t\left|\int_{t}^{\infty} u^{\lambda} d \mu(u)\right| \\
& \leqq \int_{0}^{\infty} t^{x-\lambda-1} d t \int_{t}^{\infty} u^{\lambda}|d \mu(u)| \\
& =\int_{0}^{\infty} u^{\lambda}|d \mu(u)| \int_{0}^{u} t^{x-\lambda-1} d t=\frac{1}{x-\lambda} \int_{0}^{\infty} u^{x}|d \mu(u)| \\
& \leqq K M(x-4) \quad(x \geqq 4) .
\end{aligned}
$$

When $\lambda-1<x<\lambda+1$, we have that

$$
\begin{array}{rlrl}
\int_{0}^{\infty} t^{x}\left|d \nu_{n}(t)\right| & \leqq \int_{0}^{\infty} t^{x-2}\left|d \nu_{n}(t)\right|+\int_{0}^{\infty} t^{x+2}\left|d \nu_{n}(t)\right| \\
& \leqq K M(x-6)+K M(x-2) & & (x \geqq 6) \\
& \leqq 2 K M(x-2) & & (x \geqq 6) .
\end{array}
$$

On combining these results, we obtain that, if $x \geqq 6$,

$$
\int_{0}^{\infty} t^{x}\left|d v_{n}(t)\right| \leqq 2 K M(x-2)
$$

We now define constants $A_{p}$ by

$$
A_{p}=\int_{p}^{p+1}\left|d \nu_{n}(t)\right| \quad(p=1,2,3, \ldots) .
$$

From (3.12), we deduce that, for any given $p(0<p<\infty)$ and any $x \geqq 6$,

$$
A_{p} p^{x}=p^{x} \int_{p}^{p+1}\left|d v_{n}(t)\right| \leqq \int_{0}^{\infty} t^{x}\left|d v_{n}(t)\right| \leqq 2 K M(x-2)
$$

Hence,

$$
\frac{A_{p} p^{x}}{2 K} \leqq M(x-2)=\max _{t \geqq 0}\left|\frac{t^{x-2}}{H_{0}(t)}\right|
$$

Therefore,

$$
\log \frac{A_{p} p^{x}}{2 K} \leqq \max _{s}\left\{s x-\left(h_{0}(s)+2 s\right)\right\}=k(x),
$$

say. But $h_{0}(s)+2 s$ is a convex function of $s$, and thus, by (3.9),

$$
h_{0}(s)+2 s=\max _{s}\{s x-k(x)\} \text {. }
$$

We may write this in the form

$$
\log t^{2} H_{0}(t)=\max _{x}\{x \log t-k(x)\} .
$$

Thus

$$
t^{2} H_{0}(t) \leqq \max _{x}\left\{t^{x} \exp (-k(x))\right\} \leqq \max _{x}\left\{\frac{2 K}{A_{p}}\left(\frac{t}{p}\right)^{x}\right\}
$$


by (3.13) provided that the maximum value of $x \log t-k(x)$ is assumed for a value of $x \geqq 6$. This will be the case as long as $t$ is large enough-say $t \geqq p_{0}$, where $p_{0}$ is a constant which depends only on the function $H(s)$.

We choose $t=p \geqq p_{0}$ in (3.14) and obtain that, for $p \geqq p_{0}, p^{2} H_{0}(p) \leqq 2 K / A_{p}$. Hence

$$
A_{p} H(p+1) \leqq 2 K / p^{2} \quad\left(p \geqq p_{0}\right) .
$$

We therefore conclude that

$$
\begin{aligned}
\left\|\nu_{n}\right\|_{H} & =\int_{0}^{\infty} H(t)\left|d v_{n}(t)\right| \\
& \leqq H\left(p_{0}\right) \int_{0}^{\infty}\left|d \nu_{n}(t)\right|+\sum_{p=p_{0}}^{\infty} A_{p} H(p+1) \\
& \leqq H\left(p_{0}\right) \int_{0}^{\infty}|d \mu(t)|+2 K \sum_{p=p_{0}}^{\infty} \frac{1}{p^{2}}=A,
\end{aligned}
$$

where $A$ is a constant which depends only on the function $H(s)$ and on $\mu$. This completes the proof of Lemma 1.

Suppose now that $V$ is not dense in $S_{H}$. Then it follows from Lemma 1 that the sequence $\left\{x^{\lambda_{n}}\right\}$ is free in $S_{H}$. Hence the linear functional $L_{n}$ defined on $V$ by (3.6) is bounded. Its extension $\bar{L}_{n}$ as a bounded linear functional to $\bar{V}$ is therefore unique. Given any $f \in \bar{V}$, we may therefore define $a_{n}=\bar{L}_{n}(f)$ and write

$$
f(x) \sim \sum a_{n} x^{\lambda_{n}}
$$

This definition says nothing about the convergence of the series in (3.15). However, if

$$
P_{n}(x)=\sum a_{n k} x^{\lambda_{k}}
$$

is a polynomial with the property that $\left\|P_{n}-f\right\|_{H} \rightarrow 0(n \rightarrow \infty)$, then $a_{n k} \rightarrow a_{k}$ $(n \rightarrow \infty)$ for each $k=1,2, \ldots$.

LEMMA 2. Suppose that $V$ is not dense in $S_{H}$. Then, given any $f \in \bar{V}$ for which $f(x) \sim \sum a_{n} x^{\lambda_{n}}$, we have that

$$
\left|a_{n}\right| \leqq C^{\lambda_{n}} \exp \left\{-2 \lambda_{n} \sum_{k=1}^{n} \lambda_{k}^{-1}\right\} \cdot\|f\|_{H}
$$

where $C$ is a constant depending only on the set $\Lambda$ and on $H(s)$.

Proof. In view of the continuity of $\bar{L}_{n}(n=1,2, \ldots)$, we need prove this only in the case when $f$ is a polynomial $P \in V$. We have

$$
a_{n}=\int_{0}^{\infty} P(t) d \mu_{n}(t),
$$

where the measure $\mu_{n}$ is that of Lemma 1 . Hence 


$$
\begin{aligned}
\left|a_{n}\right| & \leqq \max _{t \geqq 0}\left|\frac{P(t)}{H(t)}\right| \cdot \int_{0}^{\infty} H(t)\left|d \mu_{n}(t)\right| \\
& =\|P\|_{H} \cdot\left\|\mu_{n}\right\|_{H} \\
& \leqq C^{\lambda_{n}} \exp \left\{-2 \lambda_{n} \sum_{k=1}^{n} \lambda_{k}^{-1}\right\} \cdot\|P\|_{H}
\end{aligned}
$$

by Lemma 1, as required.

4. Problems of closure. Suppose now that $V$ is not dense in $S_{H}$. Then how big is the closure $\bar{V}$ of $V$ in $S_{H}$ ? We have already considered the case when $\sum \lambda_{n}^{-1}<\infty$ in the introduction. For this section we shall therefore make the assumption that

$$
\sum \lambda_{n}^{-1}=\infty
$$

We recall the definitions of $M(r)=M(r, f), m(s)$ and $\lambda(r)$ given in $\$ \$ 1$ and 3 , and define

$$
\psi(s)=\max _{n \geqq 0}\{n s-n \lambda(n)\}
$$

We now state

THEOREM 3. Suppose that (4.1) holds, and that $V$ is not dense in $S_{H}$. Then each $f \in \bar{V}$ is the restriction to the real axis of an entire function $f(z)$ with a gap power series expansion of the form

$$
f(z)=\sum A_{n} z^{\lambda_{n}}
$$

Moreover, there is a constant $a$, which depends only on the set $\Lambda$ and on $H(s)$, such that $m(s, f) \leqq \psi(s+a)$ for all sufficiently large values of $s$.

Proof. We write $C_{n}=C^{\lambda_{n}} \exp \left\{-2 \lambda_{n} \sum_{k=1}^{n} \lambda_{k}^{-1}\right\}$, and suppose that $f(x) \sim \sum A_{n} x^{\lambda_{n}}$. We then have from Lemma 2 that

$$
\left|A_{n}\right| \leqq C_{n}\|f\|_{H}
$$

Since $C_{n}^{1 / \lambda_{n}} \rightarrow 0$, we deduce that the series

$$
g(z)=\sum A_{n} z^{\lambda_{n}}
$$

converges for all values of $z$. The function $g(z)$ is therefore entire. We prove that $f(x)=g(x)(0 \leqq x<\infty)$.

Let

$$
P_{k}(x)=\sum_{n=0}^{k} A_{n k} x^{\lambda_{n}}
$$

be such that

$$
\left\|f-P_{k}\right\|_{H} \rightarrow 0 \quad(k \rightarrow \infty) .
$$


Then

$$
\left|A_{n}-A_{n k}\right| \leqq C_{n}\left\|f-P_{k}\right\|_{H}
$$

by Lemma 2. Thus, for a given $x(0 \leqq x<\infty)$,

$$
\begin{aligned}
|f(x)-g(x)| & \leqq\left|f(x)-P_{k}(x)\right|+\left|P_{k}(x)-g(x)\right| \\
& \leqq H(x)\left\|f-P_{k}\right\|_{H}+\left|\sum_{n=0}^{k}\left(A_{n k}-A_{n}\right) x^{\lambda_{n}}\right|+\left|\sum_{n=k+1}^{\infty} A_{n} x^{\lambda_{n}}\right| \\
& \leqq H(x)\left\|f-P_{k}\right\|_{H}+\left\{\sum_{n=0}^{\infty} C_{n} x^{\lambda_{n}}\right\}\left\|f-P_{k}\right\|_{H}+\left|\sum_{n=k+1}^{\infty} A_{n} x^{\lambda_{n}}\right| \\
& \rightarrow 0 \quad(k \rightarrow \infty) .
\end{aligned}
$$

Hence $f(x)=g(x)(0 \leqq x<\infty)$. Moreover,

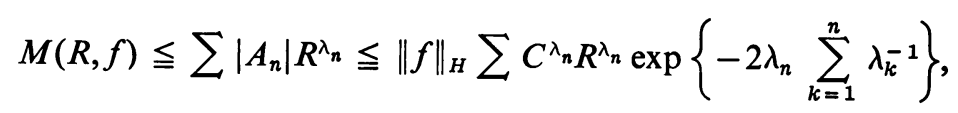

by Lemma 2 . Hence, for a suitable constant $K$,

$$
M(R, f) \leqq K \max _{n}\left\{(2 C R)^{n} \exp (-n \lambda(n))\right\} \cdot \sum 2^{-k} .
$$

On taking logarithms, we obtain that, for all large $s$,

$$
m(s, f) \leqq \max _{n}\{s n+a n-n \lambda(n)\}=\psi(s+a),
$$

where $R=e^{s}$ and $a>\log 2 C$. This completes the proof of Theorem 3 .

THEOREM 4. Let $f(z)$ be an entire function satisfying (4.3) and for which

$$
m(s, f) \leqq h(s-a)
$$

for some $a>0$ and all large values of s. Then the partial sums of the power series expansion of $f(z)$ converge to $f(z)$ in the $\|\cdot\|_{H}$ norm and hence $f \in \bar{V}$.

Proof. Let $f(z)=\sum a_{n} z^{n}$ where $a_{n}=0(n \notin \Lambda)$. It suffices to show that

$$
\left\|\sum_{n=N}^{\infty} a_{n} x^{n}\right\|_{H} \rightarrow 0 \quad(N \rightarrow \infty) .
$$

We deduce this from the fact that

$$
A=\sum_{n=0}^{\infty}\left|a_{n}\right| \sup _{t \geqq 0}\left\{\frac{t^{n}}{H(t)}\right\}<\infty .
$$

To obtain this last inequality, we begin with an estimate of the coefficients. From Cauchy's coefficient inequality,

$$
\log \left|a_{n}\right| \leqq-\sup _{s}\{n s-m(s)\}=-\sup _{t}\{n t-m(t+a)\}-n a .
$$


Writing $m_{a}(t)=m(t+a)$, we have that

$$
\left|a_{n}\right| \leqq \exp \left\{-n a-\tau_{m_{a}}(n)\right\}
$$

where, for a convex function $g, \tau_{g}$ is defined by (3.8). Also,

$$
\sup _{t \geqq 0}\left\{\frac{t^{n}}{H(t)}\right\}=\exp \left\{\sup _{s}[\operatorname{sn}-h(s)]\right\}=\exp \tau_{h}(n) .
$$

Hence

$$
A \leqq \sum_{n=0}^{\infty} \exp \left\{-n a-\tau_{m_{a}}(n)+\tau_{h}(n)\right\}
$$

A sufficient condition that $A$ be finite is therefore that $\tau_{h}(n) \leqq \tau_{m_{a}}(n)$ for large values of $n$. But this follows from (4.5) and so the proof of Theorem 4 is complete.

5. Density conditions. If the function $\psi(s)$ is given by (4.2), we define the function $\Psi(r)$ by

$$
\psi(s)=\log \Psi\left(e^{s}\right) .
$$

For $\beta>0$ we define $F_{\beta}$ to be the set of all entire functions with a gap power series expansion of the form (4.3) for which

$$
M(r, f)=O(\Psi(\beta r)) \quad(r \rightarrow \infty) .
$$

Assembling the results of the previous section and recalling the definition of $E_{\alpha}$ given in $\S 1$, we conclude that, if $\sum \lambda_{n}^{-1}=\infty$ and $V$ is not dense in $S_{H}$, then

$$
E_{\alpha} \subset \bar{V} \subset F_{\beta}
$$

for each $\alpha<1$ and some $\beta>1$. This result is less satisfactory than (1.11) which was obtained for the case $\sum \lambda_{n}^{-1}<\infty$.

A more elegant conclusion than (5.2) is possible if one is willing to admit some side conditions. For $\rho>0$ we let $G_{\rho}$ denote the set of all entire functions with a gap power series expansion of the form (4.3) and which have $h$-order at most equal to $\rho$. With this definition we have

THEOREM 5. Suppose that $\log h(s)$ is convex and that

$$
D_{h}^{*}(\Lambda)<\frac{1}{2} \text {. }
$$

Then, provided that $0<\alpha<1, G_{\alpha} \subset \bar{V} \subset G_{1}$.

We require two lemmas.

LEMMA 3. Suppose that $\log h(s)$ is a convex function of $s$. Then $V$ is dense in $S_{H}$ if $\bar{D}_{h}(\Lambda)>\frac{1}{2}$ and $V$ is not dense in $S_{H}$ if $\bar{D}_{h}(\Lambda)<\frac{1}{2}$.

Proof. The proof simply uses the criteria of Theorem B. We suppose firstly that $\bar{D}_{h}(\Lambda)>\frac{1}{2}$ and that $a$ is a given positive number. 
Then there exists a sequence of real numbers $\left\{s_{k}\right\}$ which is unbounded above and has the property that

$$
\lambda\left(s_{k}\right)-a \geqq h^{-1}\left(s_{k}\right) \quad(k=1,2, \ldots)
$$

where $\lambda(s)$ is defined by (3.1). We may assume that $s_{k}>2 s_{k-1}$ and so

$$
\int^{\infty} h(\lambda(s)-a) \frac{d s}{s^{2}} \geqq \sum^{\infty} \int_{s_{k}}^{2 s_{k}} h(\lambda(s)-a) \frac{d s}{s^{2}} \geqq \sum^{\infty} \frac{1}{2}=+\infty
$$

It follows from Theorem B that $V$ is dense in $S_{H}$.

Now suppose that $\bar{D}_{h}<\frac{1}{2}$. Then there exists a $\delta<1$ such that, for large values of $s$, $\lambda(s) \leqq \delta h^{-1}(s)$. Since $\log h(s)$ is convex, we have that

$$
h(\delta s)=O(h(s))^{\delta} \quad(s \rightarrow+\infty) .
$$

For an appropriate constant $A$,

$$
\int^{+\infty} h(\lambda(s)) \frac{d s}{s^{2}} \leqq \int^{+\infty} h\left(\delta h^{-1}(s)\right) \frac{d s}{s^{2}} \leqq A \int^{+\infty} \frac{d s}{s^{2-\delta}}<\infty .
$$

It follows from Theorem B that $V$ is not dense in $S_{H}$.

Lemma 4. Suppose that $g(x)$ is a strictly increasing function of $x$ which has a positive second derivative at every point and which satisfies (3.7). Then, if $\log g(x)$ is convex, there is a constant $\delta>0$ such that

$$
x g^{-1}(x)-x \leqq \tau_{g}(x) \leqq x g^{-1}(\delta x)
$$

for all sufficiently large values of $x$.

Proof. The left-hand inequality is obtained by substituting $x=g^{-1}(y)$ into (3.8). To prove the other inequality, we let $k(x)=g^{\prime}(x)$. Then

$$
\tau_{g}(x)=x k^{-1}(x)-g\left(k^{-1}(x)\right) \leqq x k^{-1}(x) .
$$

Since $\log g(x)$ is convex, its derivative is increasing. It follows that there is a $\delta>0$ such that

$$
\frac{d}{d x} \log g(x)=\frac{g^{\prime}(x)}{g(x)} \geqq \frac{1}{\delta}
$$

for all sufficiently large values of $x$. We let $y=g(x)$ and conclude that

$$
y / \delta \leqq g^{\prime}(x)=k\left(g^{-1}(y)\right) \text {. }
$$

Hence $g^{-1}(\delta y) \geqq k^{-1}(y)$ and the result follows from (5.5).

Proof of Theorem 5. The conclusion that $G_{\alpha} \subset \bar{V}$ for each $\alpha(0<\alpha<1)$ follows immediately from the fact that $E_{\alpha} \subset \bar{V}$ for each $\alpha(0<\alpha<1)$. It remains to show that $\bar{V} \subset G_{1}$. In view of (1.11) we may assume that $\sum \lambda_{n}^{-1}=\infty$.

Let $\Delta$ satisfy $0<\Delta<1$. By (5.3) we may embed $\Lambda$ in a set of positive integers $\mu$ for which $D_{h}(\mu)$ exists and satisfies 


$$
\frac{1}{2} \Delta<D_{h}(\mu)<\frac{1}{2} .
$$

We denote by $W$ the set of all finite linear combinations of the monomials $\left\{x^{\mu_{n}}\right\}$ and write

$$
\mu(x)=2 \sum_{\mu_{n}<x} \mu_{n}^{-1} .
$$

Evidently $\bar{V} \subset \bar{W}$. It follows from (5.6) that $\bar{D}_{h}(\mu)<\frac{1}{2}$ and therefore, by Lemma 3 , we may deduce that $W$ is not dense in $S_{H}$.

Now suppose that $f \in \bar{V}$. Then $f \in \bar{W}$ and hence, by Theorem $4, f$ is an entire function with a gap power series expansion of the form $f(z)=\sum_{n=0}^{\infty} a_{n} z^{n}$ in which $a_{n}=0(n \notin \mu)$. Further, there exists a constant $a$ such that, for large values of $s$, $m(s, f) \leqq \psi(s+a)$ where

$$
\psi(s)=\max _{n \geqq 0}\{n s-n \mu(n)\} .
$$

By (5.6) we have that $\mu(n)>\Delta h^{-1}(n)$, for all sufficiently large values of $n$. But $\log h(s)$ is convex and hence, by Lemma 4 , there is a constant $\delta$ such that $h^{-1}(n) \geqq(\delta / n) \tau_{h}(n / \delta)$. Thus, for all sufficiently large values of $n$,

$$
\mu(n)>\frac{\Delta \delta}{n} \tau_{h}\left(\frac{n}{\delta}\right) .
$$

On substituting this inequality into (5.7) and writing $n=m \delta$, we obtain that, for all large values of $s$,

$$
\begin{aligned}
\psi(s) & \leqq \max _{m \geqq 0}\left\{m \delta s-\Delta \delta \tau_{h}(m)\right\} \\
& =\Delta \delta \max _{m \geqq 0}\left\{\frac{m s}{\Delta}-\tau_{h}(m)\right\}=\Delta \delta h\left(\frac{s}{\Delta}\right)
\end{aligned}
$$

by (3.9). Thus given any $\Delta^{\prime}$ satisfying $0<\Delta^{\prime}<\Delta$ we conclude that, for large values of $s, m(s, f) \leqq h\left(s / \Delta^{\prime}\right)$. Hence

$$
\limsup _{s \rightarrow \infty} \frac{h^{-1}(m(s))}{s} \leqq \frac{1}{\Delta^{\prime}}
$$

But $\Delta$, and hence $\Delta^{\prime}$ can be chosen as close to 1 as we like. It follows then that $f(z)$ has $h$-order at most 1 ; i.e. that $\bar{V} \subset G_{1}$, as required.

6. Growth on a ray.

Proof of Theorem 1. We may suppose that $f(0)=0$. It is then a simple consequence of Theorem 1 of [1] that, for any fixed $K>1$, there is an $N$ such that

$$
\left|a_{n} r^{n}\right| \leqq K^{n} \max _{0 \leqq x \leqq r}|f(x)|
$$

for each $n \geqq N$ and every $r>0$. (For the necessary estimation of the product $\Pi(n)$ see, for example, [6].) Given $\sigma>1$, we suppose that $\sigma>K>1$. Then, for appropriate constants $B$ and $C$, 


$$
\begin{aligned}
M(r, f) & \leqq B r^{N}+\sum_{n=N}^{\infty}\left|a_{n} r^{n} \sigma^{n}\right| \sigma^{-n} \\
& \leqq B r^{N}+H(r \sigma) \sum_{n=N}^{\infty}\left(\frac{K}{\sigma}\right)^{n} \\
& =B r^{N}+C H(r \sigma)
\end{aligned}
$$

from which the theorem follows by (1.4).

Proof of Theorem 2. We may assume in the first place that $k(s) / s \rightarrow+\infty$ $(s \rightarrow+\infty)$ since Theorem A covers the case when this does not hold.

We may also assume that $\rho<1$ and that $D_{n}^{*}(\Lambda)<\frac{1}{2}$. (If necessary, we replace $h(s)$ by $h(\tau s)$ where $\tau$ is chosen so that $\tau>\rho$ and $\tau D_{h}^{*}(\Lambda)<\frac{1}{2}$.) Each of the functions $f(R t)$, where $R>0$, then has $h$-order $\rho<1$ and so we deduce from Theorem 6 that $f(R t) \in \bar{V}$ for each $R>0$.

Since the functions $h(s)$ and $k(s)$ are comparable, the equation (2.3) holds for some $l(0 \leqq l \leqq \infty)$. Since $\sigma<\rho$, we have that $l<\infty$. We distinguish two cases: when $l=0$ and when $0<l<\infty$. In view of the remark following (2.3), no loss of generality is involved in the second case if we take $h(s)=k(s)$.

Now $D_{h}^{*}(\Lambda)<\frac{1}{2}$. Hence, if $0<\Delta<1$, we may follow the argument of Theorem 6 and embed $\Lambda$ in a set of positive integers $\mu$ for which $D_{h}(\mu)$ exists and such that $\frac{1}{2} \Delta<D_{h}(\mu)<\frac{1}{2}$. With the notation of Theorem 6 we have that $W$ is not dense in $S_{H}$ and $f(R t) \in \bar{V} \subset \bar{W}$ for each $R>0$.

Now suppose that $f$ has the power series expansion (1.1). It follows from Lemma 2 that, for each $R>0$,

$$
\left|a_{n} R^{n}\right| \leqq C^{n} \exp \{-n \mu(n)\} \cdot\|f(R t)\|_{H}
$$

where $\mu$ is given by (5.5).

We now use the hypothesis that $f$ has $k$-order $\sigma$ on a ray. We can assume without loss of generality that the ray is the positive real axis. If $\sigma<\tau<1$, we obtain that, for large values of $s, \log \left|f\left(e^{s}\right)\right| \leqq k(\tau s)$. Hence, writing $R=e^{r}$, we have that

$$
\log \|f(R t)\|_{H} \leqq \sup _{s}\{k(\tau r+\tau s)-h(s)\} \text {. }
$$

Since $k(s)$ is convex,

$$
k(\tau r+\tau s) \leqq \alpha k(\tau r / \alpha)+\beta k(\tau s / \beta),
$$

provided that $\alpha$ and $\beta$ are positive and that $\alpha+\beta=1$. Thus

$$
\log \|f(R t)\|_{H} \leqq \alpha k(\tau r / \alpha)+\sup _{s}\{\beta k(\tau s / \beta)-h(s)\} .
$$

Suppose in the first instance that $l=0$. Then for any $\beta>0$,

$$
\beta k(\tau s / \beta)<h(s)
$$

for large values of $s$. Hence, for a suitable constant $a$,

$$
\log \|f(R t)\|_{H} \leqq \alpha k(\tau r / \alpha)+a .
$$


Since $\mu(n) \rightarrow \infty(n \rightarrow \infty)$, it follows from (6.1) that

$$
\left|a_{n} R^{n}\right| \leqq 2^{-n} \cdot B \exp \{\alpha k(\tau r / \alpha)\}
$$

for a suitable constant $B$. Thus

$$
M(R, f) \leqq \sum\left|a_{n}\right| R^{n} \leqq B \exp (\alpha k(\tau r / \alpha)) .
$$

Hence, if $b>0$, the inequality

$$
m(r, f) \leqq \alpha k(\tau r / \alpha)+\log B \leqq k(\tau r / \alpha+b)
$$

holds for all large values of $r$. But equation (6.3) holds for each $\beta>0$. Since $\alpha+\beta=1$, $\alpha$ may be chosen arbitrarily close to 1 . As $\tau$ is any number which satisfies $\sigma<\tau<1$, it follows that $f$ has $k$-order $\sigma$ in the whole plane. Thus the theorem is proved in the case $l=0$.

Suppose now that $0<l<\infty$. For this case we may assume that $h(s)=k(s)$. We now take $\beta=\tau$, in which case $\alpha=1-\tau$. From (6.2) it then follows that

$$
\log \|f(R t)\|_{H} \leqq \alpha k(\tau r / \alpha) .
$$

Since $\log h(s)$ is convex, we may estimate $\mu(s)$ as in Theorem 6 . We obtain that for large values of $s$,

$$
s \mu(s)>\Delta \tau_{h}(s)=\Delta \tau_{k}(s) .
$$

On substituting this result and the inequality (6.4) into (6.1), we deduce that, for a suitable constant $\gamma$,

$$
\log \left|a_{n} R^{n}\right| \leqq \gamma n-\Delta \tau(n)+\alpha k(\tau r / \alpha)
$$

Thus,

$$
\begin{aligned}
\log \left|a_{n}\right| & \leqq \gamma n-\Delta \tau_{k}(n)-\sup _{r}\{n r-\alpha k(\tau r / \alpha)\} \\
& =\gamma n-\Delta \tau_{k}(n)-\alpha \sup _{s}\{n s / \tau-k(s)\} \\
& =\gamma n-\Delta \tau_{k}(n)-\alpha \tau_{k}(n / \tau) \\
& \leqq \gamma n-(\Delta+\alpha / \tau) \tau_{k}(n)
\end{aligned}
$$

since $\tau_{k}$ is convex. It follows that

$$
\begin{aligned}
\log \left|a_{n} R^{n}\right| & \leqq-n+\sup _{n}\left\{(r+\gamma+1) n-(\Delta+\alpha / \tau) \tau_{k}(n)\right\} \\
& =-n+(\Delta+\alpha / \tau) k\left(\frac{r+\gamma+1}{\Delta+\alpha \tau^{-1}}\right) .
\end{aligned}
$$

Hence, proceeding as in the case $l=0$, we conclude that, for an appropriate constant $b, m(r, f) \leqq k(\delta r+b)$, where $\delta=(\Delta+\alpha / \tau)^{-1}$, for all sufficiently large values of $r$. In this inequality $\alpha=1-\tau$ and $\Delta$ may be chosen as close to 1 as we please. It follows that

$$
\limsup _{r \rightarrow \infty} \frac{k^{-1}(m(r))}{r} \leqq \delta
$$


where $\delta$ may be taken as close to $\tau$, and hence as close to $\sigma$ as we choose. Thus $f(z)$ has $k$-order at most $\sigma$, and the proof of Theorem 2 is complete.

7. Growth in an angle. Edrei [4] and Malliavin [11, p. 233] have both independently obtained the following result.

THEOREM C. Let $f(z)$ have order $\rho<\infty$ in some angle of opening greater than $2 \pi D_{\text {exp }}^{*}(\Lambda)$. Then $f(z)$ has order $\rho$ in the whole plane.

Malliavin deduces this from his Theorem 10.4 which is a consequence of his approximation results. His method also yields the following theorem. In this theorem the number $D^{\dagger}(\Lambda)$ is defined by

$$
D^{\dagger}(\Lambda)=\limsup _{x / y \rightarrow+\infty} \frac{L(x)-L(y)}{\log x-\log y} .
$$

THEOREM 6. Let $f(z)$ have h-order $\rho<\infty$ in some angle of opening greater than $2 \pi D^{\dagger}(\Lambda)$. Then $f(z)$ has $h$-order $\rho$ in the whole plane.

Malliavin calls the number $D^{\dagger}(\Lambda)$ the quasi-logarithmic density of $\Lambda$. As he remarks

$$
\bar{D}_{\text {exp }}(\Lambda) \leqq D_{\text {exp }}^{*}(\Lambda) \leqq D^{\dagger}(\Lambda),
$$

and, therefore, Theorem 6 is weaker than Theorem $\mathrm{C}$ in the case when $h$ is the exponential function. It would be interesting to know whether Theorem 6 remains true in general with $D^{\dagger}(\Lambda)$ replaced by $D_{\text {exp }}^{*}(\Lambda)$.

Theorem 6 is a consequence of Theorem $\mathrm{D}$ below. This is simply a version of Malliavin's Theorem 10.4. Before quoting Theorem $D$, we require a few definitions.

If $k(r)$ is a real valued function on $(0, \infty)$, the function $k_{0}(r)$ is defined by

$$
k_{0}(r)=\inf _{s \geqq r} k(s) .
$$

The function $k(r)$ is said to be asymptotically increasing if the function $k(r)-k_{0}(r)$ is bounded $(0<r<\infty)$.

The quasi-logarithmic density $D^{\dagger}(\Lambda)$, defined by (7.1) above, turns out to be the greatest lower bound $\left({ }^{2}\right)$ of the set of all $\alpha$ for which $\alpha \log x-L(x)$ is asymptotically increasing.

The function $g(z)$ will be assumed to be analytic at the origin with a gap power series expansion of the form

$$
g(z)=\sum_{n=0}^{\infty} c_{n} z^{n}
$$

in which $c_{n}=0(n \notin \Lambda)$.

(2) Not the least upper bound as in [11, p. 218], which is, presumably, a misprint. 
THEOREM D. Let $\mathscr{S}$ denote the closure of a sector of the unit disc which subtends an angle $\gamma>2 \pi \bar{D}_{\exp }(\Lambda)$ at the origin. Suppose that $g(z)$, which is given by (7.3) in the neighborhood of the origin, is continuous on $\mathscr{S}$ and analytic on its interior. Then

$$
\left|c_{n}\right| \leqq \exp \left\{\alpha+\beta n+\left(k(n)-k_{0}(n)\right) n\right\} \max _{z \in \mathscr{S}}|g(z)|,
$$

where $\alpha$ and $\beta$ are positive constants which depend only on the set $\Lambda$ and the sector $\mathscr{S}$ and $k(x)=(\gamma / \pi) \log x-\lambda(x)$.

Theorem 6 is now readily proved. If $\gamma>2 \pi D^{\dagger}(\Lambda)$ then the function $k(x)$ is asymptotically increasing-i.e. $k(n)-k_{0}(n)$ is bounded. In view of (7.2) we may apply Theorem D to the function $g(z)=f(R z)$ for an arbitrary value of $R>0$. We obtain that, for an appropriate constant $a$,

$$
M(a R, f) \leqq \max _{z \in \mathscr{S}}|f(R z)| \quad(R>0) .
$$

The assumption that $f(z)$ has $h$-order $\rho$ in an angle of opening $\gamma$ then yields the required result. Theorem $\mathrm{C}$ is proved similarly (see [11, p. 233]).

\section{REFERENCES}

1. J. M. Anderson and K. G. Binmore, Coefficient estimates for lacunary power series and Dirichlet. I, Proc. London Math. Soc. (3) 18 (1968), 36-48. MR 36 \#6624.

2. - On entire functions with gap power series, Glasgow Math. J. (to appear).

3. J. A. Clarkson and P. Erdös, Approximation by polynomials, Duke Math. J. 10 (1943), 5-11. MR 4, 196.

4. A. Edrei, Gap and density theorems for entire functions, Scripta Math. 23 (1958), 117-141. MR 20 \#5281.

5. W. H. J. Fuchs, On the closure of $\left\{e^{-t} t^{a_{v}}\right\}$, Proc. Cambridge Philos. Soc. 42 (1946), 91-105. MR 7, 294.

6. D. Gaier, On the coefficients and the growth of gap power series, SIAM J. Numer. Anal. 3 (1966), 248-265. MR 34 \#4492.

7. J.-P. Kahane, Travaux de Beurling et Malliavin, Séminaire Bourbaki 1961/62, Exposé 225 Secrétariat mathématique, Paris, 1962. MR 26 \#3561; errata, MR 30 p. 1203.

8. J. Korevaar, $A$ characterisation of the sub-manifold of $C[a, b]$ spanned by the sequence $\left\{x^{n} k\right\}$, Nederl. Akad. Wetensch. Proc. Ser. A 50 (1947), 750-758=Indag. Math. 9 (1947), 360-368. MR 9, 346.

9. T. Kövari, On the growth of entire functions of finite order with density conditions, Quart.

J. Math. Oxford Ser. (2) 17 (1966), 22-30. MR 33 \#1456.

10. A. F. Leont'ev, On the completeness of a system of powers on a semi-axis, Izv. Akad. Nauk SSSR Ser. Mat. 26 (1962), 781-792. (Russian) MR 26 \#4080.

11. P. Malliavin, Sur quelques procédés d'extrapolation, Acta Math. 93 (1955), 179-255. MR 17, 724.

12. S. Mandelbrojt, Séries adhérentes, régularisation des suites, applications, GauthierVillars, Paris, 1952. MR 14, 542.

University COLlege of LONDON, LoNdon, ENGLAND

LONDON SCHOOL OF ECONOMICS, LONDON, ENGLAND 